\title{
PREVALENCE AND ANTIBIOTIC SENSITIVITY PATTERN OF STAPHYLOCOCCUS AUREUS FROM ALL CLINICAL SAMPLES WITH EMPHASIS ON MRSA IN A TERTIARY CARE HOSPITAL
}

\author{
Avneet Kaur Heyar'1, Amarjit Kaur Gill², Anchal Mahajan³, Kamaldeep Kaur ${ }^{4}$ \\ ${ }_{1}^{1}$ Research Scholar, Department of Microbiology, Centre for Interdisciplinary Biomedical Research, Adesh University, Bathinda, Punjab. \\ 2 Professor and HOD, Department of Microbiology, Adesh Institute of Medical Sciences and Research, Bathinda, Punjab. \\ ${ }^{3}$ Assistant Professor, Department of Microbiology, Adesh Institute of Medical Sciences and Research, Bathinda, Punjab. \\ ${ }^{4}$ Research Scholar, Department of Microbiology, Centre for Interdisciplinary Biomedical Research, Adesh University, Bathinda, Punjab.
}

ABSTRACT

\section{BACKGROUND}

S. aureus is a versatile pathogen that causes human diseases ranging from mild infections to life-threatening sepsis. Therefore, to prevent $S$. aureus infections, effective infection control practices and antibiotic policies should be formulated. The present study was conducted to study the prevalence and antibiotic sensitivity pattern of Staphylococcus aureus (S. aureus) isolated from various clinical samples in a tertiary care hospital.

\section{MATERIAL AND METHODS}

A descriptive study was conducted in Microbiology Department. In the present study, out of 1194 clinical isolates, 206 S. aureus isolates were identified and processed using standard microbiology procedures. The antibiotic susceptibility test was performed by Kirby-Bauer disc diffusion method as per CLSI guidelines.

\section{RESULTS}

The prevalence of S. aureus was reported as 206 (17.25\%) and MRSA was reported as 99 (48.0\%). High resistance was seen in case of Penicillin whereas resistance less than 50\% was reported in Methicillin, Ciprofloxacin, Gentamicin, Erythromycin; and Clindamycin. Vancomycin, Teicoplanin and Linezolid were found to be $100 \%$ sensitive.

\section{CONCLUSION}

S. aureus is a common cause of skin and soft tissue infections in hospitals. There should be a regular surveillance on the changing patterns of antibiotic sensitivity pattern of MRSA and antibiotic policy should be formulated to reduce the increasing levels of infections in the hospitals.

\section{KEYWORDS}

Staphylococcus aureus, MRSA, Antibiotic Sensitivity Pattern.

HOW TO CITE THIS ARTICLE: Heyar AK, Gill AK, Mahajan A, et al. Prevalence and antibiotic sensitivity pattern of staphylococcus aureus from all clinical samples with emphasis on MRSA in a tertiary care hospital. J. Evolution Med. Dent. Sci. 2017;6(84):58575860, DOI: $10.14260 /$ jemds/2017/1272

\section{BACKGROUND}

Staphylococcus aureus is recognised as a causative agent of nosocomial and community-acquired infections in almost every region of the world.[1] Staphylococci are the leading pathogens which are known to cause skin and soft tissue infections (SSTIs).[2] Antibiotic resistance in S. aureus has become an increasing problem among outpatients, hospitalised patients and even in health care persons. ${ }^{[3]}$

S. aureus are Gram-positive cocci approximately $1 \mu \mathrm{m}$ in diameter and are arranged in grape like clusters. It expresses many potential virulence factors which include production and secretion of toxins and enzymes; alpha, beta, gamma and delta toxins act on the cell membrane of host and mediates cell destruction. Some exotoxins like exfoliative, toxic shock syndrome toxin (TSST) and some enterotoxins are also produced.

'Financial or Other Competing Interest': None.

Submission 12-09-2017, Peer Review 06-10-2017,

Acceptance 12-10-2017, Published 19-10-2017.

Corresponding Author:

Avneet Kaur Heyar,

\#195, Model Town,

Phase III, Bathinda,

Punjab-151001, India.

E-mail: heyar.avneet@gmail.com

DOI: $10.14260 /$ jemds $/ 2017 / 1272$
Clumping factor, coagulase and hyaluronidase enhances invasion and its survival in the tissues. ${ }^{[4]}$

In humans, Staphylococcus aureus causes a variety of suppurative (pus-forming) infections. It causes skin and soft tissue infections such as furuncles (boils), folliculitis, abscess (particularly breast abscess), wound infections; infections such as pneumonia, meningitis and urinary tract infections; musculoskeletal infections such as osteomyelitis, arthritis and endovascular infections such as bacteraemia, septicaemia, endocarditis. S. aureus also causes hospitalacquired (nosocomial) infections that are associated with medical indwelling devices and surgical wounds. It also releases enterotoxins that causes food poisoning and also cause toxic shock syndrome by releasing superantigens into the blood stream. ${ }^{[4]}$

The emergence of resistance to antimicrobial agents among staphylococci was increasing. Then introduction of penicillin in 1941 tackled the problem. In 1961, Methicillinresistant Staphylococcus aureus (MRSA) was first reported and since then, it has become a major nosocomial pathogen. ${ }^{[5,6]}$ Methicillin resistance in $S$. aureus is mediated by production of altered penicillin binding protein 2a (PBP-2a) which is encoded by a gene called mecA.[7]

The glycopeptide antibiotic vancomycin was first released in 1958, as the drug of choice for MRSA. Subsequently, 
vancomycin has been used for treatment of serious infections caused by MRSA for many years. Initial reports of reduced susceptibility to vancomycin in S. aureus from Japan in 1997 from various clinical samples generated significant concern in the medical community. ${ }^{[8]}$ There is renewed interest in the use of Macrolide-Lincosamide-Streptogramin $B \quad\left(M L S_{B}\right)$ antibiotics due to increase in frequency of Staphylococcal infections and change in patterns of antimicrobial resistance..$^{[9]}$

The aim of the present study was to determine the antibiotic sensitivity pattern of strains of $S$. aureus isolated from various clinical samples.

\section{MATERIALS AND METHODS}

A descriptive study was conducted in Department of Microbiology, AIMSR, Bathinda, Punjab. All clinical samples like pus, sputum, urine, blood, genital specimens (high vaginal swab, semen), body fluids (cerebrospinal fluid, pleural fluid, synovial fluid, etc.), devices (endotracheal tube, tracheostomy tube, drain, etc.) were included in the study.

The samples were collected under aseptic conditions and were further processed and identified using standard microbiological procedures. The specimens were cultured on the Nutrient Agar (NA), Blood Agar (BA) and MacConkey Agar (MA), incubated overnight at $37^{\circ} \mathrm{C}$ in incubator. [10]

Gram staining was performed on colonies that were suggestive of $S$. aureus by colony morphology followed by microscopy. The samples showing Gram-positive cocci in clusters were processed further for confirmation by biochemical reactions whether the organisms were $S$. aureus. Tests like catalase, slide coagulase and tube coagulase were carried as confirmatory tests for S. aureus. ${ }^{[10]}$

The antibiotic susceptibility testing was done on MuellerHinton Agar by Kirby-Bauer disk diffusion method. A 0.5 McFarland turbidity inoculum was prepared by sub-culturing 45 colonies in nutrient broth and incubated at $37^{\circ} \mathrm{C}$ for $4 \mathrm{hrs}$. in incubator. Lawn culture was made on $\mathrm{MH}$ agar and antibiotic sensitivity of isolates was checked using penicillin $(10 \mu \mathrm{g})$, cefoxitin $(30 \mu \mathrm{g})$, cephalexin $(30 \mu \mathrm{g})$, cefuroxime $(30 \mu \mathrm{g})$, cefotaxime $(30 \mu \mathrm{g})$, amoxicillin + clavulanic acid $(20 / 10 \mu \mathrm{g})$, gentamicin $(10 \mu \mathrm{g})$, ciprofloxacin $(5 \mu \mathrm{g})$, azithromycin $(15 \mu \mathrm{g})$, erythromycin $(15 \mu \mathrm{g})$, clindamycin $(2 \mu \mathrm{g})$, vancomycin $(30 \mu \mathrm{g})$, teicoplanin $(30 \mu \mathrm{g})$ and linezolid $(15 \mu \mathrm{g})$ as per CLSI guidelines.[11] The culture plates were then incubated at $37^{\circ} \mathrm{C}$ for 24 hrs. in incubator.

\section{RESULTS}

In the present study which was carried out in microbiology lab, AIMSR, out of 1194 clinical isolates, 206 were S. aureus and the prevalence of $S$. aureus from all clinical samples being
$17.25 \%$. Out of the 206, MRSA were 99 (48\%) and MSSA were 107 (51.9\%) as shown in Table I.

\begin{tabular}{|c|c|}
\hline Isolate & Prevalence \\
\hline MRSA & $48 \%(99)$ \\
\hline MSSA & $52 \%(107)$ \\
\hline Table I. Prevalence of MRSA and MSSA \\
\hline
\end{tabular}

Out of 206 S. aureus isolates, 101(49.0\%) were from OPD, $83(40.3 \%)$ were from IPD and $22(10.7 \%)$ were from ICU as shown in Table II.

\begin{tabular}{|c|c|c|c|}
\hline Department & $\begin{array}{c}\text { \% Isolation } \\
\text { (n=206) }\end{array}$ & $\begin{array}{c}\text { \% age of MRSA } \\
\text { in each Dept. }\end{array}$ & $\begin{array}{c}\text { \% of MSSA in } \\
\text { each Dept. }\end{array}$ \\
\hline OPD & $49.0 \%(101)$ & $48.5 \%(49)$ & $51.5 \%(52)$ \\
\hline IPD & $40.3 \%(83)$ & $48.2 \%(40)$ & $51.8 \%(43)$ \\
\hline ICU & $10.7 \%(22)$ & $45.5 \%(10)$ & $54.5 \%(12)$ \\
\hline \multicolumn{4}{|c|}{ Table II. Prevalence of S. aureus from Various } \\
Departments \\
\hline
\end{tabular}

Out of 206 S. aureus isolates, the percentage isolation from urine was $33.4 \%$, from pus $45.1 \%$, in blood $4.8 \%$, sputum $2.4 \%$, body fluids $3.3 \%$ and from various devices $10.6 \%$ as shown in Table III. Maximum number of $S$. aureus isolates were from pus samples.

\begin{tabular}{|c|c|c|c|}
\hline Specimens & $\begin{array}{c}\text { Percentage of } \\
\text { Age Isolation } \\
\text { of } \text { S. aureus } \\
(\mathbf{n = 2 0 6 )}\end{array}$ & $\begin{array}{c}\text { Percentage } \\
\text { Isolation of } \\
\text { MRSA in each } \\
\text { Specimen }\end{array}$ & $\begin{array}{c}\text { Percentage } \\
\text { Isolation of } \\
\text { MSSA in Each } \\
\text { Specimen }\end{array}$ \\
\hline Urine & $33.4 \%(69)$ & $50.7 \%(35)$ & $49.3 \%(34)$ \\
\hline Pus & $45.1 \%(93)$ & $47.4 \%(44)$ & $52.6 \%(49)$ \\
\hline Blood & $4.8 \%(10)$ & $40 \%(4)$ & $60 \%(6)$ \\
\hline Sputum & $2.4 \%(5)$ & $100 \%(5)$ & 0 \\
\hline Body fluid & $3.3 \%(7)$ & $28.5 \%(2)$ & $71.5 \%(5)$ \\
\hline Devices & $10.6 \%(22)$ & $41 \%(9)$ & $59 \%(13)$ \\
\hline \multicolumn{4}{|c|}{$\begin{array}{c}\text { Table III. Prevalence of S. aureus } \\
\text { from Various Clinical Samples }\end{array}$} \\
\hline \multicolumn{4}{|c|}{} \\
\hline
\end{tabular}

The antibiotic resistance pattern of all 206 S. aureus isolates towards following antibiotics was: Penicillin 178 (86.4\%), Cefoxitin 99 (48.05\%), Cephalexin 99 (48.05\%), Cefuroxime 99 (48.05\%), Amoxicillin + Clavulanic Acid 99 (48.05\%), Gentamicin 75 (36.40\%), Ciprofloxacin 83 (40.29\%), Levofloxacin 27 (13.10\%), Azithromycin 29 (14.07\%), Erythromycin 67 (32.52\%), Clindamycin 36 $(17.47 \%)$ and there were no strains which were found resistant to Vancomycin, Teicoplanin and Linezolid as shown in Table IV.

\begin{tabular}{|c|c|c|c|c|}
\hline Antibiotic & Resistance & Sensitive & $\begin{array}{c}\text { Susceptibility pattern of } \\
\text { MRSA strains }\end{array}$ & $\begin{array}{c}\text { Susceptibility pattern of MSSA } \\
\text { strains }\end{array}$ \\
\hline Penicillin & $86.4 \%(178)$ & $213.6 \%(28)$ & 0 & $26.2 \%(28)$ \\
\hline Cefoxitin & $48.1 \%(99)$ & $51.9 \%(107)$ & 0 & $100 \%$ \\
\hline Cephalexin & $48.1 \%(99)$ & $51.9 \%(107)$ & 0 & $100 \%$ \\
\hline Cefuroxime & $48.1 \%(99)$ & $51.9 \%(107)$ & 0 & $100 \%$ \\
\hline Cefotaxime & $48.1 \%(99)$ & $51.9 \%(107)$ & 0 & $100 \%$ \\
\hline Amoxicillin+ Clavulanic Acid & $48.1 \%(99)$ & $51.9 \%(107)$ & 0 & $100 \%$ \\
\hline Gentamicin & $36.4 \%(75)$ & $63.6 \%(131)$ & $51.5 \%(51)$ & $74.8 \%(80)$ \\
\hline Ciprofloxacin & $40.3 \%(83)$ & $59.7 \%(123)$ & $27.3 \%(27)$ & $89.7 \%(96)$ \\
\hline
\end{tabular}




\begin{tabular}{|c|c|c|c|c|}
\hline Azithromycin & $14.1 \%(29)$ & $15.9 \%(177)$ & $82.8 \%(82)$ & $88.8 \%(95)$ \\
\hline Erythromycin & $32.5 \%(67)$ & $67.5 \%(139)$ & $40.4 \%(40)$ & $92.5 \%(99)$ \\
\hline Clindamycin & $17.5 \%(36)$ & $82.5 \%(170)$ & $77.8 \%(77)$ & $86.9 \%(93)$ \\
\hline Vancomycin & 0 & $100 \%$ & $100 \%$ & $100 \%$ \\
\hline Teicoplanin & 0 & $100 \%$ & $100 \%$ & $100 \%$ \\
\hline Linezolid & 0 & $100 \%$ & $100 \%$ & $100 \%$ \\
\hline \multicolumn{7}{|c|}{ Table IV. Prevalence of Antibiotic Susceptibility Pattern } \\
\hline
\end{tabular}

\section{DISCUSSION}

Among the Gram-positive pathogens, S. aureus is a common cause of skin and soft tissue infection in community and as well as hospitalised patients. The prevalence of $S$. aureus in the present study was $17.25 \%$ from all clinical samples. Other studies reported prevalence as $6 \%, 24.5 \%, 42.73 \%$ and $48.14 \% \cdot[12,13,14,15]$

In the present study, the prevalence of $S$. aureus isolates in OPD, IPD and ICU was 49.0\%, 40.3\% and $10.7 \%$ respectively. The prevalence of MRSA was $48.5 \%$ in OPD, $48.2 \%$ in IPD and $45.5 \%$ in ICU. In a study conducted by INSAR group, India, the prevalence of MRSA was 28.4\%, $42.3 \%$ and $43.6 \%$ from OPD, IPD and ICU respectively in 2008 and $27 \%, 49 \%$ and $47 \%$ from OPD, IPU and ICU respectively in 2009.[16] The increased rate of isolation from OPD could be because our institute is a tertiary care hospital where patients are referred after prior treatment failure and over-the-counter empirical treatment.

The prevalence of $S$. aureus from various clinical specimens in this study were $33.4 \%$ from urine, $45.1 \%$ from pus, $4.8 \%$ in blood, $2.4 \%$ in sputum, $3.3 \%$ in body fluids and $10.6 \%$ from devices. In another two studies, the prevalence of pus was maximum i.e. $39.48 \%[17]$ and $68.7 \% .[15]$ Thus, our study is in accordance to most other studies where prevalence of $S$. aureus is maximum from pus sample.

The prevalence of MRSA in this study from various specimens was $50.7 \%$ in urine, $47.4 \%$ in pus, $40 \%$ in blood, $100 \%$ in sputum, $28.5 \%$ in body fluids and $41 \%$ in devices. In a study conducted INSAR group, India the prevalence was $41 \%, 36 \%, 44 \%$ and $34 \%$ in urine, pus, blood and body fluids respectively in 2008. Whereas in 2009 the prevalence showed a slight change and the results found were $52 \%, 40 \%$, $48 \%, 54 \%$ in urine, pus, blood and body fluids respectively.[16]

Cefoxitin is the indicator drug for methicillin-resistant $S$. aureus by disc diffusion method as per the CLSI guidelines.[11] All isolates that are Cefoxitin resistant are also resistant to all Penicillins, Cephalosporins, Beta lactam group + Beta lactamases inhibitor combination and carbapenems. Hence, overall prevalence of MRSA in this study was found to be $48.1 \%$. Our study is in accordance with the study conducted in Jaipur by Gupta et al in 2015 which showed MRSA prevalence of $40 \%{ }^{[18]}$ Similarly researches conducted in Benin, Bengaluru South East Nigeria showed a prevalence similar to the ongoing study.[12,15,13]

A study was conducted by Indian network for Surveillance of Antimicrobial Resistance (INSAR) group, India in different leading hospitals of India to study the prevalence and susceptibility pattern of MRSA. The overall prevalence observed was $42 \%$ in 2008 and $40 \%$ in 2009 for MRSA.[16]

The variation may be due to difference in the methods of detection, infection control practices and usage of antibiotics which vary from hospital to hospital. This could be the reason of non-uniform epidemiology of MRSA in India.[18] Very low prevalence of $5.26 \%$ and $6 \%$ were seen in studies from Peshawar in 2014[19] and Benin in 2011[12] respectively.

In our study, 178 (86.4\%) isolates out of 206 were resistant to Penicillin. A similar prevalence rate of resistance against Penicillin was observed in studies from South East Nigeria[13] and Jaipur.[17] Dhanalakshmi TA et al showed 85.6\% S. aureus isolates were resistant to Penicillin.[20] In our study, all the MRSA strains were reported resistant to Penicillin whereas, $73.8 \%$ MSSA strains were resistant and $26.2 \%$ were sensitive. All the $S$. aureus isolates in the present study were sensitive to Vancomycin, Teicoplanin and Linezolid. This finding is similar to the studies conducted by Arora et al in 2010[21] and Sharma $M$ et al in 2013[17] where Vancomycin and Linezolid were found to be $100 \%$ sensitive. Our study showed $36.4 \%$ resistance towards Gentamicin which was similar to a study conducted in South East Nigeria in 2016 by Chijioke A Nsofor et al where Gentamicin resistance was $31.7 \% .^{[13]}$ Two other similar studies conducted by Dhanalakshmi TA et al [20] and Sina $\mathrm{H}$ et al [12] showed $33.6 \%$ and $42.27 \%$ resistance respectively towards Gentamicin. On the other hand, in present study, the resistance pattern for Ciprofloxacin was found to be $40.3 \%$. Similar results were seen in studies from South East Nigeria[13] and Jaipur, ${ }^{[17]}$ where the resistance was $38.5 \%$ and $41.53 \%$ respectively.

In case of macrolides that include Azithromycin and Erythromycin, the resistance in our study was found to be considerably low. Azithromycin resistance was $14.1 \%$ in the present study whereas in other studies the prevalence was $32.8 \%[20]$ and $45.12 \%{ }^{[17]}$ that is comparatively higher than our study. In case of Erythromycin, the resistance in our study was $32.5 \%$. A study from Benin has reported resistance similar to our study i.e. $35.77 \%$.[12] Some other researches have reported high resistance against Erythromycin.[13,17,20,21] In the ongoing study, 59.6\% MRSA isolates and 7.5\% MSSA isolates showed resistance against erythromycin. A study carried out by INSAR group, India reported higher resistance in MRSA and MSSA isolates.[16]

In the present study, the prevalence of resistance against Clindamycin was $17.5 \%$ among $S$. aureus isolates. Two other studies showed a similar resistance pattern, Dhanalakshmi TA et al[20] reported $14 \%$ resistance and Sharma M et al[17] reported $9.72 \%$. The prevalence of resistance in our study was $22.2 \%$ in MRSA isolates and $13.1 \%$ in MSSA. In a study conducted by INSAR group, India, the prevalence of resistance was reported as $46.6 \%$ and $14.7 \%$ in MRSA and MSSA isolates respectively.[16] 


\section{CONCLUSION}

In conclusion, adequate preventive and control measures should be taken to reduce MRSA infections by formulating antibiotic policies and effective infection control practices.

The progressive increase in positivity of MRSA in $S$. aureus isolates calls for routine testing of methicillin resistance by using Cefoxitin disc diffusion method or Minimum Inhibitory Concentration (MIC) detection method. Vancomycin, Teicoplanin and Linezolid are still the drugs of choice for MRSA infections. Thus, there is a need of in vitro susceptibility testing for all the isolated clinical samples for selection of drugs that are appropriate for treatment of the infection, thereby limiting the overdose of powerful antibiotics which might help in changing the trends of antibiotic susceptibility pattern.

\section{REFERENCES}

[1] Yilmaz G, Aydin K, Iskender S, et al. Detection and prevalence of inducible clindamycin resistance in staphylococci. J Med Microbiol 2007;56(Pt 3):342-5.

[2] Angel MR, Balaji V, Prakash J, et al. Prevalence of inducible clindamycin resistance in gram positive organisms in a tertiary care centre. Indian J Med Microbiol 2008;26(3):262-4.

[3] Lewis JS, Jorgensen JH. Inducible clindamycin resistance in Staphylococci: should clinicians and microbiologists be concerned? Clin Infect Dis 2005;40(2):280-5.

[4] Tille PM. Bailey and Scott's diagnostic microbiology. $13^{\text {th }}$ edn. Missouri: Elsevier, 2014:232-8.

[5] Mallick SK, Basak S, Bose S. Inducible clindamycin resistance in Staphylococcus aureus-a therapeutic challenge. Journal of Clinical and Diagnostic Research 2009;3(3):1513-8.

[6] Brown DF, Edwards DI, Hawkey PM, et al. Guidelines for the laboratory diagnosis and susceptibility testing of methicillin resistant Staphylococcus aureus (MRSA). J Antimicrob Chemother 2005;56(6):1000-18.

[7] Bressler AM, Williams T, Culler EE, et al. Correlation of penicillin binding protein 2a detection with oxacillin resistance in Staphylococcus aureus and discovery of a novel penicillin binding protein 2 a mutation. J Clin Microbiol 2005;43(9):4541-4.

[8] Howden BP, Davies JK, Johnson PD, et al. Reduced vancomycin susceptibility in Staphylococcus aureus, including vancomycin-intermediate and heterogeneous vancomycin-intermediate strains: resistance mechanisms, laboratory detection and clinical implications. Clin Microbiol Rev 2010;23(1):99-139.

[9] Fiebelkorn KR, Crawford SA, McElmeel ML, et al. Practical disc diffusion method for detection of inducible clindamycin resistance in Staphylococcus aureus and coagulase negative Staphylococci. J Clin Microbiol 2003;41(10):4740-4.
[10] Collee JG, Fraser AG, Marmion BP, et al. Mackie and McCartney practical medical microbiology. $14^{\text {th }}$ edn. Delhi: Elsevier, 1999:246-9.

[11] Clinical and laboratory Standards Institute. Performance standards for antimicrobial susceptibility testing: seventeenth informational supplement. 2007;27(1).

[12] Sina H, Baba-Moussa F, Ahoyo TA, et al. Antibiotic susceptibility and toxins production of Staphylococcus aureus isolated from clinical samples from Benin. African J Micro Research 2011;5(18):2797-803.

[13] Nsofor CA, Nwokenkwo VN, Ohale CU. Prevalence and antibiotic susceptibility pattern of Staphylococcus aureus isolated from various clinical specimens in south east Nigeria. MOJ Cell Sci Rep 2016;3(2):1-5.

[14] Singh MP, Sharma SK, Shukla S, et al. Prevalence rate and antibiotic susceptibility test (AST) pattern of methicillin resistant Staphylococcus aureus (MRSA) isolates from different clinical specimens of Teerthankar Mahaveer Hospital, Moradabad, India. International J Current Micro and Applied Sci 2013;2(11):307-14.

[15] Debnath A, Chikkaswamy BK. Antibiogram and susceptibility pattern of methicillin-resistant Staphylococcus aureus collected from various clinical samples in Bengaluru. Asian J Pharm Clin Res 2015;8(6):260-4.

[16] Joshi S, Ray P, Manchanda V, et al. Methicillin resistant Staphylococcus aureus (MRSA) in India: prevalence and susceptibility pattern. Indian J Med Res 2013;137(2):363-9.

[17] Sharma M, Pathak S, Srivastava P. Prevalence and antibiogram of methicillin resistance Staphylococcus aureus at a tertiary care hospital in Jaipur, Rajasthan. International Journal of Pharmaceutical Research and Bio-Science 2013;2(1):139-47.

[18] Gupta S, Dongre A, Pandey AC, et al. Antibiogram of methicillin resistant Staphylococcus aureus (MRSA) in healthcare settings. J Chemical and Pharm Research 2015;7(8):61-6.

[19] Khan RA, Rahman AU, Ahmad A, et al. Prevalence and antibiotic susceptibility profile of methicillin-resistant Staphylococcus aureus (MRSA) isolated from different clinical samples in district Peshawar. J Appl Environ Biol Sci 2014;4(8S):40-6.

[20] Dhanalakshmi TA, Umapathy BL, Mohan DR. Prevalence of methicillin, vancomycin and multidrug resistance among Staphylococcus aureus. J Clinical and Diag Research 2012;6(6):974-7.

[21] Arora S, Devi P, Arora U, et al. Prevalence of methicillin resistant Staphylococcus aureus (MRSA) in a tertiary care hospital in Northern India. J Lab Physicians 2010;2(2):78-81. 